\title{
INSUFFICIENCY OF TORRES' CONDITIONS FOR TWO-COMPONENT CLASSICAL LINKS
}

\author{
M. L. PLATT
}

\begin{abstract}
Torres has given necessary conditions for a polynomial to be the Alexander polynomial of a two component link. For certain links, additional conditions are necessary. Hillman gave one example for linking number 6 . Here we give examples for all other linking numbers except $0, \pm 1$, and \pm 2 .
\end{abstract}

1. Introduction. In 1953, Torres [T] gave necessary conditions for a polynomial to be the Alexander polynomial of a link. More recently, in the case of two component links with linking number $b$, Bailey [B] showed equivalently that the Alexander polynomial of the link can be expressed in the form

$$
\Delta(x, y)=\frac{1-(x y)^{b}}{1-x y} A(x, y)-(1-x)(1-y)\left(\frac{1-(x y)^{b-1}}{1-x y}\right) B(x, y),
$$

where $A(x, y)$ and $B(x, y)$ satisfy certain conditions.

Using Bailey's result, Hillman $[\mathbf{H}]$ gave an additional condition on the Alexander polynomial of certain two component links whose linking number is divisible by at least two distinct primes. In $\S 3$ of this paper, a similar result is given for prime power linking numbers in

(3.7) THEOREM. Let $L$ be a two-component link with linking number, $p^{\alpha}$, where $p$ is a prime. Let $\lambda(x)=a\left(x+x^{-1}\right)+(1-2 a)$, where $\lambda(-1)$ is square-free, and let $\Delta(x, y)$ be the Alexander polynomial of $L$. If the knot polynomial

$$
(1-x)^{-1}\left(1-x^{p^{\alpha}}\right) \Delta(x, 1)=\lambda(x)
$$

and if $\omega$ is a primitive $p^{\beta}$ th root of unity for some $\beta \leqslant \alpha$, then the $\mathbf{Z}[\omega] / \lambda(\omega)$-ideal generated by $B(\omega, 1)(\bmod \lambda(\omega))$ is of the form $J \bar{J}$ for some ideal $J$.

It should be noted that the ideal $J \bar{J}$ depends only on $\Delta(x, y)$ and not on the expansion given above.

Following (3.7), we show how to realize counterexamples to Torres' condition for two-component links, provided the linking number of the components is not $0, \pm 1$, or \pm 2 . It should be noted that the Torres conditions do suffice if $b=0$ or \pm 1 . Hence, only the case when $b=2$ remains unsettled. Finally, a counterexample to Torres' conditions for $m$-component links $(m>3)$ is given.

Received by the editors June 23, 1985 and, in revised form, September 19, 1985.

1980 Mathematics Subject Classification (1985 Revision). Primary 57M25: Secondary 57M10. 
This paper is a revised version of part of the author's Ph.D. thesis written under the guidance of Jerome P. Levine, to whom we would like to express our deep gratitude. We are also grateful to Paul Monsky, who made many helpful suggestions.

2. Definitions. A classical link of multiplicity $m$ is a collection, $L=L_{1} \cup \cdots \cup L_{m}$ of oriented smooth simple closed curves in $S^{3}$ satisfying $L_{i} \cap L_{j}=\varnothing$ if $i \neq j$. The number $m$ denotes the number of components of the link. If $m=1$, then $L$ is a knot. The number $b=\operatorname{lk}\left(L_{i}, L_{j}\right)$ is the linking number of the $i$ th component and the $j$ th component. A link is trivial if it is the boundary of $m$ disjoint 2-disks in $S^{3}$.

The complement of the link is the space

$$
X=S^{3}-\bigcup_{i=1}^{m} \nu\left(L_{i}\right),
$$

where $\nu\left(L_{i}\right)$ is a small open tubular neighborhood of $L_{i}$. The neighborhoods $\nu\left(L_{i}\right)$ can be chosen so small that $\nu\left(L_{i}\right) \cap \nu\left(L_{j}\right)=\varnothing$ if $i \neq j$. The basepoint of $X$ is denoted by $*$.

For each $i$, let $m_{i}$ be a small circle linking the $i$ th component $L_{i}$ of $L$ with $\operatorname{lk}\left(m_{i}, L_{i}\right)=1$, let $l_{i}$ be a translate of $L_{i}$ into $X$ whose basepoint coincides with that of $m_{i}$ and such that $\operatorname{lk}\left(l_{i}, L_{i}\right)=0$ and let $\gamma_{i}$ be a path in $X$ from $*$ to the basepoint of $m_{i}$. The elements $\alpha_{i}$ of $\pi_{1} X$ represented by $\gamma_{i}^{*} m_{i}^{*} \gamma_{i}^{-1}$ are called meridians of the link. The elements $\beta_{i}$ of $\pi_{1} X$ represented by $\gamma_{i}^{*} l_{i}^{*} \gamma_{i}^{-1}$ are called longitudes of the link. The pair $\left(\alpha_{i}, \beta_{i}\right)$ is determined up to simultaneous conjugation by the element of $\pi_{1} X$.

An orientation of a link consists of an ordering of the components together with an orientation of each component.

By Alexander duality, $H_{1}(X) \cong \mathbf{Z}^{m}$, where $m$ is the multiplcity of the link. A canonical basis of $H_{1}(X)$, defined by any choice of meridians of $L$, allow the identification of $\mathbf{Z}\left[H_{1}(X)\right]$ with $\Lambda_{m}=\mathbf{Z}\left[t_{1}, t_{1}^{-1}, \ldots, t_{m}, t_{m}^{-1}\right]$, the identification depending only on the orientation of the link. There is a natural involution of $\Lambda_{m}$ (denoted with an overbar) which maps $t_{i} \rightarrow t_{i}^{-1}$. The augmentation of $\Lambda_{m}$ is given by $\varepsilon: \Lambda_{m} \rightarrow \mathbf{Z}$, where $\varepsilon\left(t_{i}\right)=1$.

The canonical homomorphism $h: \pi_{1} X \rightarrow H_{1}(X)$ defines a regular covering space $p: \tilde{X} \rightarrow X$ with $\mathbf{Z}^{m}$ as the group of covering transformations. The space $\tilde{X}$ is called the universal abelian cover of $X$. The Alexander module of $L$ is $H_{1}(\tilde{X}, \tilde{*})$ considered as a module over $\Lambda_{m}$. The module of $L$ is $H_{1}(\tilde{X})$ considered as a module over $\Lambda_{m}$.

These modules are related by the exact sequence

$$
0 \rightarrow H_{1}(\tilde{X}) \rightarrow H_{1}(\tilde{X}, \tilde{*}) \stackrel{\Phi}{\rightarrow} M \rightarrow 0,
$$

where $\Phi$ is the boundary homomorphism, $\Phi: H_{1}(\tilde{X}, \tilde{*}) \rightarrow H_{0}(\tilde{*})$, and $M$ is the augmentation ideal of $\Lambda_{m}$ generated by $t_{1}-1, \ldots, t_{m}-1$ [L-3].

Given a presentation matrix for $H_{1}(\tilde{X}, \tilde{*})$ as a $\Lambda_{m}$-module, the sequence of elementary ideals, or Fitting invariants, is $\tilde{E}_{i}(L)$, where $\tilde{E}_{i}(L)$ is the ideal of $\Lambda_{m}$ generated by the $(n-i)$-order minors of the presentation matrix [F]. Let $\tilde{\Delta}_{i}(L)$ be 
the greatest common divisor or $\tilde{E}_{i}(L)$. A sequence of ideals $E_{i}(L)$ and polynomials $\Delta_{i}(L)$ can be defined the same way from a presentation matrix for $H_{1}(\tilde{X})$. Although $\tilde{E}_{i}(L) \neq E_{i-1}(L)$ in general, $\tilde{\Delta}_{i}(L)=\Delta_{i-1}(L)[\mathbf{L}-\mathbf{1}]$. The Alexander polynomial of $L$ is

$$
\tilde{\Delta}_{1}(L)=\Delta_{L}\left(t_{1}, \ldots, t_{m}\right)
$$

\section{Two-component links.}

A. Suppose $L=K_{1} \cup K_{2}$ is a two-component link with linking number $b$. Under these circumstances Torres [T] has shown that the Alexander polynomial of $L$ can be chosen to have the following properties:

$$
\text { (1) } \begin{aligned}
\Delta(x, y) & =x^{b-1} y^{b-1} \Delta\left(x^{-1}, y^{-1}\right), \\
\text { (2) } \Delta(x, 1) & =\left(1-x^{b}\right)(1-x)^{-1} \Delta_{2}(x), \\
\Delta(1, y) & =\left(1-y^{b}\right)(1-y)^{-1} \Delta_{1}(y),
\end{aligned}
$$

where $\Delta_{2}(x)$ and $\Delta_{1}(y)$ are knot polynomials. In fact, $\Delta_{2}(x)$ and $\Delta_{1}(y)$ are the Alexander polynomials of the component knots corresponding to the meridians $x$ and $y$, respectively.

Bailey [B] has characterized the module of $L, H_{1}(\tilde{X})$, as a $\Lambda_{2}$-module having a presentation matrix with a certain symmetry condition. Bailey's main result is the following theorem.

(3.2) Theorem (BAiley). A $\Lambda_{2}$-module is a link module if and only if it has a presentation matrix of the form

$$
M_{l .}=\left[\begin{array}{cc}
\left(1-(x y)^{h}\right)(1-x y)^{-1} & -\left[(1-x)(1-y)\left(1-(x y)^{b-1}\right)(1-x y)^{-1} \beta(x, y)\right] \\
\beta^{\operatorname{tr}}\left(x^{-1}, y^{-1}\right) & \mathbf{A}(x, y)
\end{array}\right]
$$

where $\beta(x, y)$ is a row matrix, $\mathbf{A}(x, y)$ is a square matrix with entries in $\Lambda_{2}$, satisfying $\mathbf{A}(x, y)=\mathbf{A}^{\mathrm{tr}}\left(x^{-1}, y^{-1}\right)$ and $\mathbf{A}(1,1)=\operatorname{diag}( \pm 1, \ldots, \pm 1)$. Furthermore, $\mathbf{A}(x, 1)$ (resp. $\mathbf{A}(1, y))$ is a presentation matrix for the first (resp. second) component of the link and $b$ is the linking number of the components.

One corollary of Bailey's theorem is that the Alexander polynomial of a two-component link has the form

$$
\begin{aligned}
D(x, y)= & \left(1-(x y)^{h}\right)(1-x y)^{-1} A(x, y) \\
& -(1-x)(1-y)(1-x y)^{-1}\left(1-(x y)^{h-1}\right) B(x, y),
\end{aligned}
$$

where $A(x, y)=A\left(x^{-1}, y^{-1}\right), B(x, y)=B\left(x^{-1}, y^{-1}\right)$, and $A(x, 1)$ and $A(1, y)$ are knot polynomials.

For instance, one may take

$$
A(x, y)=\operatorname{det} \mathbf{A}(x, y), \quad B(x, y)=\operatorname{det}\left[\begin{array}{cc}
0 & \beta(x, y) \\
\beta^{\operatorname{tr}}\left(x^{-1}, y^{-1}\right) & \mathbf{A}(x, y)
\end{array}\right] .
$$


Moreover, Bailey showed that a polynomial in $\Lambda_{2}$ has this form if and only if it satisfies (3.1).

Using Bailey's result, Hillman has proven the following theorem.

(3.4) Theorem (Hillman). Let $L$ be a two-component link with linking number $b>1$ and with Alexander polynomial $\Delta(x, y)$. If the knot polynomial $\left(1-x^{b}\right)$ $\cdot(1-x)^{-1} \Delta(x, 1)$ is (up to units) the d-cyclotomic polynomial, $\Phi_{d}(x)$, for some $d>1$ dividing $b$ and if $\omega$ is a primitive dth root of unity, then the $\mathbf{Z}[\omega]$-ideal generated by $B(\omega, 1)$ is of the form $J \bar{J}$ for some $J$.

The hypothesis of the theorem is vacuous unless $d$ is divisible by at least two distinct primes. Hillman's theorem suggests two questions:

(3.5) Question 1. Do counterexamples to (3.1) exist whenever $b$ is a nonprime power number?

(3.6) Question 2. Do counterexamples to (3.1) exist if $b$ is a power of a prime?

B. To answer (3.5), suppose $d$ is a nonprime power number, $\Phi_{d}(x)$ is the $d$-cyclotomic polynomial and $a$ is an integer. Let

$$
\begin{aligned}
D(x, y)= & \left(1-(x y)^{d}\right)(1-x y)^{-1} \Phi_{d}(x) \\
& -(1-x)(1-y)\left(1-(x y)^{d-1}\right)(1-x y)^{-1}(a) .
\end{aligned}
$$

By direct computation, one finds that $\Phi_{d}(x)$ is a knot polynomial; hence $D(x, y)$ satisfies (3.1). If $\omega$ is a primitive $d$ th root of unity for $d \neq p^{\alpha}$, one may ask, in view of Hillman's theorem, if there is an integer, $a$, such that the ideal generated by $a$ does not factor as $J \bar{J}$ in $\mathbf{Z}[\omega]$ ?

Suppose that $q$ is a prime, $q+d$, and $Q$ is a prime of $\mathbf{Z}[\omega]$ lying over $q$. The prime $q$ is unramified since the only ramified primes are those dividing $d$. The Galois group, $\operatorname{Gal}(\mathbf{Q}[\omega] / \mathbf{Q})$ is isomorphic to $(\mathbf{Z} / d)^{\times}$. The decomposition group of $q$, $D(Q \mid q)$, is the (cyclic) subgroup of $\operatorname{Gal}(\mathbf{Q}[\omega] / \mathbf{Q})$ generated by $\omega \rightarrow \omega^{q}$, which corresponds to the subgroup of $(\mathbf{Z} / d)^{\times}$generated by $q$.

Suppose that complex conjugation, $\sigma$, is an element of the decomposition group, in other words, that $Q=\bar{Q}$. This will happen, for instance, if $q \equiv-1(\bmod d)$, and by Dirichlet's density theorem there are infinitely many such primes. Now, any such prime $q$ factors in $\mathbf{Z}[\omega]$ as $\Pi Q_{i}$ with each $Q_{i}$ distinct and $Q_{i}=\bar{Q}_{i}$. In particular, $(q) \neq J \bar{J}$ for any ideal $J$ of $\mathbf{Z}[\omega]$. One may then take $a=q$ and

$$
\begin{aligned}
D(x, y)= & \left(1-(x y)^{d}\right)(1-x y)^{-1} \Phi_{d}(x) \\
& -(1-x)(1-y)\left(1-(x y)^{d-1}\right)(1-x y)^{-1}(q) .
\end{aligned}
$$

C. In order to answer (3.6) one uses an argument similar to that in [H]. Suppose the linking number is a prime power, say $b=p^{\alpha}$. Suppose further that in (3.3) $A(x, 1)=\lambda(x)=a\left(x+x^{-1}\right)+(1-2 a)$ and $\lambda(-1)=1-4 a$ is square-free. Then $\lambda(x)$ is a knot polynomial and $R=\Lambda_{2} /(\lambda(x), y-1)$ is a Dedekind domain [L-2]. (Note that $R=\mathbf{Z}\left[\alpha, \alpha^{-1}\right]$, where $\alpha$ is a root of $\lambda(x)$ and that the image of $A(x, 1)$ in 
$R$ is 0 .) Let $q$ be a prime ideal of $R$ such that $q=\bar{q}$ and consider the localizations

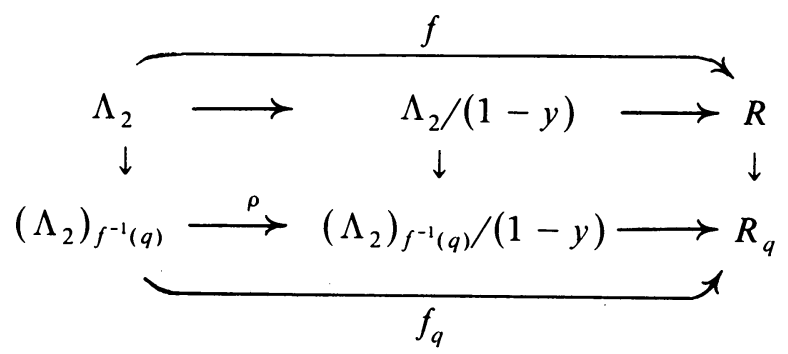

Since $f(A)=f(\operatorname{det} \mathbf{A})=0, f_{q}(A)=0 . R_{q}$ is a Euclidean domain, so the rows of $f_{q}(\mathbf{A})$ are linearly dependent. Hence, the first row of $f_{q}(\mathbf{A})$ can be reduced to zero by elementary row operations. By performing the conjugate column operations, the first column of $f_{q}(\mathbf{A})$ can be reduced to zero as well. An elementary $f_{q}$-matrix can be lifted to an elementary $\left(\Lambda_{2}\right)_{f^{-1}(q)}$-matrix, so there is an elementary $\left(\Lambda_{2}\right)_{f^{-1}(q)^{-}}$ matrix, $\mathbf{P}$, such that $f_{q}\left(\mathbf{P A} \overline{\mathbf{P}}^{\text {tr }}\right)$ has first row and column zero (here, bar denotes $x \rightarrow x^{-1}$ ). Let $\mathbf{Q}=1 \oplus \mathbf{P}$. Then $\mathbf{Q B} \overline{\mathbf{Q}}$ has the form

$$
\left[\begin{array}{ccc}
0 & \beta_{1} & \gamma \\
\bar{\beta}_{1} & a \lambda(x)+(y-1) b & \lambda(x) \mu+\nu(y-1) \\
\bar{\gamma}^{\text {tr }} & \lambda\left(x^{-1}\right) \bar{\mu}^{\text {tr }}+\left(y^{-1}-1\right) \bar{\nu}^{\text {tr }} & \mathbf{C}
\end{array}\right]
$$

where $\gamma, \mu, \nu$ are row matrices with entries in $\left(\Lambda_{2}\right)_{f^{-1}(q)}, \mathbf{C}=\overline{\mathbf{C}}^{\text {tr }}$ is a square matrix with entries in $\left(\Lambda_{2}\right)_{f^{-1}(q)}, a, b$ and $\beta_{1}$ are elements of $\left(\Lambda_{2}\right)_{f^{-1}(q)}$, and $\operatorname{ker} f_{q}=$ $(y-1, \lambda(x))$.

Since $A(x, 1)=\lambda(x), \quad \operatorname{ker} f_{q}=(y-1, \lambda(x))$ and the matrix PA $\overline{\mathbf{P}}^{\text {tr }}$ in $\left(\Lambda_{2}\right)_{f^{-1}(q)} /(y-1)$ has the form

Hence,

$$
\left[\begin{array}{cc}
a \lambda(x) & \lambda(x) \mu \\
\lambda(x) \bar{\mu}^{\operatorname{tr}} & \mathbf{C}
\end{array}\right]
$$

$$
\lambda(x)=\rho(\operatorname{det} \mathbf{A})=\rho\left(\operatorname{det} \mathbf{P A} \overline{\mathbf{P}}^{\operatorname{tr}}\right)=a \lambda(x) \rho(\operatorname{det} \mathbf{C})\left(\bmod \lambda^{2}\right) .
$$

That is,

$$
1=a \rho(\operatorname{det} \mathbf{C})(\bmod \lambda),
$$

so $f_{q}(\operatorname{det} \mathbf{C})$ is a unit in $R_{q}$. Therefore, the ideal $\left(f_{q}(\operatorname{det} \mathbf{B})\right)=\left(f_{q}\left(\beta_{1}\right)\right)\left(\overline{f_{q}\left(\beta_{1}\right)}\right)$. Since $R_{q}$ is a discrete valuation ring, let $v_{q}(I)$ be defined by $I_{q}=q^{i^{\prime} q}(I)$ for each ideal $I$ of $R$. Thus, if $q=\bar{q}, v_{q}\left(f_{q}(\operatorname{det} \mathbf{B})\right)=2 v_{q}\left(f_{q}\left(\beta_{1}\right)\right)=2 w_{q}$. If $q \neq \bar{q}, v_{q}\left(f_{q}(\operatorname{det} \mathbf{B})\right)=$ $v_{\bar{q}}\left(f_{\bar{q}}(\operatorname{det} \mathbf{B})\right)$ since $f_{q}(\operatorname{det} \mathbf{B})=f_{\bar{q}}(\operatorname{det} \mathbf{B})$ for all $q$. Let $z_{q}=v_{q}\left(f_{q}(\operatorname{det} \mathbf{B})\right)$ in this case. Let $S=\left\{q \neq \bar{q} \mid z_{q}>0\right\}$ and let $T \subset S$ contain exactly one representative of each conjugate pair. Let

$$
J=\prod_{r \in T} r^{z_{q}} \prod_{q=\bar{q}} q^{n^{*}}
$$

Then $v_{q}(J \bar{J})=v_{q}\left(f_{q}(\operatorname{det} \mathbf{B})\right)$ for all primes $q$ of $R\left(\right.$ i.e., $\left.(J \bar{J})_{q}=f_{q}(\operatorname{det} \mathbf{B}) \forall q\right)$. Thus, $f(\operatorname{det} \mathbf{B})=J \bar{J}[S]$. 
Now let $\omega$ be a primitive $p^{\beta}$ th root of unity, $\beta \leqslant \alpha\left(d=p^{\beta}\right.$ where $d$ divides $\left.b\right)$. Consider $R \stackrel{g}{\rightarrow} \mathbf{Z}[\omega] / \lambda(\omega)$, where $g$ is defined by evaluation. The ideal generated by the image of $B$ in $\mathbf{Z}[\omega] / \lambda(x)$ is of the form $J \bar{J}$ for some ideal $J$ since the involution in $\Lambda_{2}$ is compatible with complex conjugation in $\mathbf{Z}[\omega]$. Thus one has

(3.7) THEOREM. Let $L$ be a two-component link with linking number, $p^{\alpha}$, where $p$ is a prime. Let $\lambda(x)=a\left(x+x^{-1}\right)+(1-2 a)$, where $\lambda(-1)$ is square-free and let $\Delta(x, y)$ be the Alexander polynomial of $L$. If the knot polynomial

$$
(1-x)^{-1}\left(1-x^{p^{\alpha}}\right) \Delta(x, 1)=\lambda(x)
$$

and if $\omega$ is a primitive $p^{\beta}$ th root of unity for some $\beta \leqslant \alpha$, then the $\mathbf{Z}[\omega] / \lambda(\omega)$-ideal generated by $\mathbf{B}(\omega, 1)(\bmod \lambda(\omega))$ is of the form $J \bar{J}$ for some ideal $J$.

Question 2 can now be specialized as follows.

(3.8) Question 2'. Let

$$
\begin{aligned}
D(x, y)= & \left(1-(x y)^{p^{\prime \prime}}\right)(1-x y)^{-1}\left(a\left(x+x^{-1}\right)+1-2 a\right) \\
& -(1-x)(1-y)\left(1-(x y)^{p^{\prime \prime}-1}\right)(1-x y)^{-1}(c) .
\end{aligned}
$$

Is it possible to choose $a$ and $c$ so that

(i) $4 a-1$ is square-free,

(ii) c does not generate an ideal of the form $J \bar{J}$ in $\mathbf{Z}[\omega] / \lambda(\omega)$ ?

The answer to the question is yes, provided $p \neq 2$.

D. Let $\omega$ be a primitive $p$ th root of unity and let $\theta=\omega+\omega^{-1}-2$. Then $\lambda(\omega)=1+a \theta$.

Consider the diagram

$$
\begin{array}{ccc}
L=\mathbf{Q}[\omega] & \supset & \mathbf{Z}[\omega] \\
\operatorname{degree} 2 \downarrow & & \downarrow \\
K=\mathbf{Q}[\theta] & \supset & \mathbf{Z}[\theta] \\
\operatorname{degree}(p-1) / 2 \downarrow & & \downarrow \\
\mathbf{Q} & \supset & \mathbf{Z}
\end{array}
$$

The following properties are easily established $[\mathbf{L a}-\mathbf{1}, \mathbf{M}]$.

(i) $\mathbf{Z}\left[\omega+\omega^{-1}\right]=\mathbf{Z}[\theta]$.

(ii) $f(a)=N_{K / \mathbf{Q}}(\lambda(\omega))$ splits over $\mathbf{Z}[\theta]$ into factors which are linear in $a$.

(iii) $\theta=\left(\omega^{-1}-1\right)(1-\omega)$ and $N_{K / Q}(\theta)=(-1)^{[(p-1) / 2]} p$.

(iv) If $q \in \mathbf{Z}$ is a prime such that $q \equiv-1(\bmod p)$, then $q$ splits into $r=(p-1) / 2$ distinct primes in $\mathbf{Z}[\boldsymbol{\theta}]$. Furthermore, the decomposition group of $q$ is $D=\langle\boldsymbol{\sigma}\rangle$ where $\sigma$ is complex conjugation, so $Q_{i}=\bar{Q}_{i}$ for each $Q_{i}$ dividing $q$ in $\mathbf{Z}[\omega]$.

Now fix $q \equiv-1(\bmod p)$ and let $Q$ be a prime dividing $q$.

(3.9) LemmA. If $1+a \theta \in Q$, then for any $Q^{\prime}(\neq Q)$ dividing $q, 1-a \theta \notin Q^{\prime}$. Hence, if $1+a \theta \in Q^{n}$, then $1+a \theta \notin\left(Q^{\prime}\right)^{n}$.

PRoOF. If $p=3, \mathbf{Q}[\theta]=\mathbf{Q}$, and there is nothing to prove. If $p>3$, let $(q)=Q_{1}$ $\cdots Q_{(p-1) / 2}$ be the splitting of $q$ in $\mathbf{Z}[\theta]$. WLOG $Q=Q_{1}$ and $Q^{\prime}=Q_{2}$. Suppose $1+a \theta \in Q_{1}$ and $1+a \theta \in Q_{2}$. There is $\tau \in \operatorname{Gal}(\mathbf{Q}[\theta]: \mathbf{Q})$ such that $\tau\left(Q_{1}\right)=Q_{2}$. 
Now $\tau(\theta)=\theta^{\prime}$ for some $\theta \neq \theta^{\prime}$ since $\theta$ is primitive. Hence,

$$
\tau(1+a \theta)=1+a \theta^{\prime}
$$

Therefore

$$
(1+a \theta)-\left(1+a \theta^{\prime}\right)=a\left(\theta-\theta^{\prime}\right) \in Q_{2} .
$$

However, $a \notin Q_{2}$ since $a \in Q_{2}$ implies $1 \in Q_{2}$ and $\theta-\theta^{\prime} \notin Q_{2}$ since $\theta-\theta^{\prime}$ is only divisible by primes lying over $p$. This cannot happen since $Q_{2}$ is a prime ideal and $q \neq p$.

(3.10) Lemma. There is an $a \in \mathbf{Z}$ such that $1+a \theta \in Q^{2}$. Hence, $f(a) \equiv 0$ $\left(\bmod q^{2}\right)$ has an integral solution, and these conditions on a are equivalent.

Proof. $\mathbf{Z}[\theta] / Q \simeq \mathbf{Z} / q$. Let $g(a)=1+a \theta$. There is a solution to $g(a)=0$ in $\mathbf{Z}[\boldsymbol{\theta}] / Q$ since $\mathbf{Z}[\boldsymbol{\theta}] / Q$ is a field and $\boldsymbol{\theta}$ is nonzero in $\mathbf{Z}[\boldsymbol{\theta}] / Q$. For $a$, one takes the corresponding element of $\mathbf{Z} / q$. For this choice of $a, f(a) \equiv 0(\bmod q)$. If $f(a) \not \equiv 0$ $\left(\bmod q^{2}\right)$, then $a$ can be modified $(\bmod q)$ so that $f(a) \equiv 0\left(\bmod q^{2}\right)$. This follows because

$$
\begin{aligned}
f(a+k q) & \equiv f(a)+k q f^{\prime}(a) \quad\left(\bmod q^{2}\right) \\
& \equiv q r+k q f^{\prime}(a) \quad\left(\bmod q^{2}\right) \quad(\text { since } f(a) \equiv 0 \bmod q) \\
& \equiv q\left(r+k f^{\prime}(a)\right) \quad\left(\bmod q^{2}\right) .
\end{aligned}
$$

$f$ and $f^{\prime}$ are relatively prime, so $f^{\prime}(a) \not \equiv 0(\bmod q)$. Hence, one seeks $k$ such that

$$
f(a+k q) \equiv q\left(r+k f^{\prime}(a)\right) \equiv 0 \quad\left(\bmod q^{2}\right) .
$$

Equivalently,

$$
r+k f^{\prime}(a) \equiv 0 \quad(\bmod q) .
$$

But $h(k)=r+f^{\prime}(a) k$ is a linear polynomial in $k$; hence it has a root in $\mathbf{Z} / q$. Let $a^{\prime}=a+k q$. Then $g\left(a^{\prime}\right) \equiv 0\left(\bmod Q^{2}\right)$ and $f\left(a^{\prime}\right) \equiv 0\left(\bmod q^{2}\right)$. Similarly,

(3.11) LeMMA. $1-4 a \not \equiv 0(\bmod q)$.

Finally, if $1-4 a$ is not a prime, Dirichlet's density theorem allows modification of $a\left(\bmod q^{2}\right)$ so that $1-4 a$ is a prime. That is, $a$ can be chosen within a given residue class $\bmod q^{2}$ so that $1-4 a$ is prime.

In summary, for $\lambda(x)=a\left(x+x^{-1}\right)+(1-2 a)$ there is a prime $q=-1(\bmod p)$ and an integer $a$ such that $1-4 a$ is prime and such that $\lambda(\omega) \in Q^{2}$, where $Q$ is a prime in $K$ dividing $q$. All that remains is to show that $q$ does not factor as $J \bar{J}$ in $\mathbf{Z}[\omega] / \lambda(\omega)$.

The condition $(q) \neq J \bar{J}$ in $\mathbf{Z}[\omega] / \lambda(\omega)$ is equivalent to

$$
(q)+(\lambda(\omega)) \neq J \bar{J}+(\lambda(\omega)) \text { in } \mathbf{Z}[\omega] .
$$


Suppose $\lambda(\omega)=\prod Q_{i}^{e_{i}} \Pi P_{j}^{f^{\prime}}, P_{j} \neq Q_{i}$, is a factorization of $\lambda(\omega)$ in $\mathbf{Z}[\omega]$. (It is possible that some of the $e_{i}$ s are zero.) Then

$$
\begin{aligned}
& (q)+(\lambda(\omega))=\prod_{i=1}^{(p-1) / 2} Q_{i}^{\min \left(1, e_{1}\right)}, \\
& J \bar{J}+(\lambda(\omega))=\prod_{i=1}^{(p-1) / 2} Q_{i}^{h_{i}} \Pi P_{j}^{g_{1}},
\end{aligned}
$$

where $h_{i}=e_{i}$ or some even integer less than $e_{i}$. (If $Q_{i}^{h}$ is the $Q_{i}$-factor of $J$, then $Q_{i}^{2 h}$ is the $Q_{i}$-factor of $J \bar{J}$.) Thus, for inequality, it is sufficient that some $e_{i}$ be at least 2. By Lemmas 3.9 and 3.10 the first time this situation occurs is when $f(a) \equiv 0$ $\left(\bmod q^{2}\right)$.

Thus, to realize a counterexamples to (3.1) for a given linking number $b$, one needs to fix a prime $p$ dividing $b$ and consider $f(a)$, the norm of $1+a \theta$. If $f(a) \equiv 0(\bmod q)$ with $q=-1(\bmod p)$, then $a$ can be $\operatorname{modified}(\bmod q)$ so that $f(a) \equiv 0\left(\bmod q^{2}\right)$. If $1-4 a$ is not square-free, then $1-4 a$ can be modified $\left(\bmod q^{2}\right)$ so that $1-4 a$ is square-free (in fact, $1-4 a$ can be modified $\left(\bmod q^{2}\right)$ so that it is a prime). Then

$$
\begin{aligned}
D(x, y)= & \left(1-(x y)^{b}\right)(1-x y)^{-1}\left(a\left(x+x^{-1}\right)+(1-2 a)\right) \\
& -(1-x)(1-y)\left(1-(x y)^{b-1}\right)(1-x y)^{-1}(q)
\end{aligned}
$$

satisfies (3.1) but is not the Alexander polynomial for any two-component link with linking number $b$.

E. Comment on the case $p^{\alpha}=2^{\alpha}$. If $\alpha=1$, then condition (i) requires that $\lambda(-1)$ be square-free. Also, $\omega=-1$ in this case. Hence, $\lambda(\omega)=\lambda(-1)$. Condition (ii) then requires $\lambda(-1)$ to have a square factor. Hence, this technique will not yield a counterexample to (3.1) because (i) and (ii) cannot be satisfied simultaneously.

If $b=4$, then $\mathbf{Z}[\omega]=\mathbf{Z}[i]$ and Question $2^{\prime}$ (3.8) can be specialized in the following easily realizable conditions.

(i) $1-4 a$ is square free.

(ii) -1 is not a square $(\bmod q)$ and $\lambda(i)=1-2 a \equiv 0\left(\bmod q^{2}\right)$.

Condition (ii) assures that $q$ is a prime in $\mathbf{Z}[i]$. If $1-2 a \equiv 0\left(\bmod q^{2}\right)$, then the argument of the previous section shows that $(q) \neq J \bar{J}$ in $\mathbf{Z}[i] / \lambda(i)$. Hence, in (3.8), one takes $c=q$ and

$$
\begin{aligned}
D(x, y)= & \left(1-(x y)^{4}\right)(1-x y)^{-1}\left(a\left(x+x^{-1}\right)+1-2 a\right) \\
& -(1-x)(1-y)\left(1-(x y)^{3}\right)(1-x y)^{-1} \cdot q .
\end{aligned}
$$

Similar counterexamples exist for any $b=2^{\alpha}$ with $\alpha>1$.

F. Examples. The case $p=3$.

In this case $\mathbf{Q}[\boldsymbol{\theta}]=\mathbf{Q}, \mathbf{Q}[\omega]=\mathbf{Q}[\sqrt{-3}], \lambda(\omega)=1-3 a$ and $q \equiv-1(\bmod 3)$ reduces to $q$ remains prime in $\mathbf{Z}[\omega]$ (since $r=1$ ). The conditions can be reformulated as follows.

(i) $1-4 a$ is square-free.

(ii) -3 is not a square $(\bmod q)(q=2$ is excluded $)$ and $1-3 a \equiv 0\left(\bmod q^{2}\right)$. For instance, one may take $q=5$ and $a=17$. 
For $p=5, q=19$ and $a=9-19^{2}$. Then, $f(a)=19^{2} \cdot 1721$ and $1-4 a=1409$, so the conditions are met.

4. A generalization for $m$-component links. Let $B=\left(b_{i j}\right)$ be a matrix of linking number [L-1]. The entry in row $i$ and column $j, b_{i j}$, is $1 \mathrm{k}\left(L_{i}, L_{j}\right)$. The diagonal entries are undefined. If $B$ is an $(m \times m)$ matrix, a splitting, $S$, of $B$ is a proper subset of the integers $\{1, \ldots, m\}$ such that $b_{i j}=0$ whenever $i \in S$ and $j \notin S$. If there is a splitting of $B, B$ is said to be splittable. Levine [L-2] has shown that any link with an unsplittable linking matrix has nonzero Alexander polynomial and that the zero polynomial is allowed if and only if $B$ is splittable. Let $B_{i}$ denote the matrix obtained from $B$ by deleting the $i$ th row and column.

For convenience, let $b_{i i}=0$ and consider

$$
B=\left[\begin{array}{llll}
0 & b & 0 & 0 \\
b & 0 & 1 & 0 \\
0 & 1 & 0 & 1
\end{array}\right]
$$

Consider, also, the polynomial

$$
f_{m}\left(t_{1}, \ldots, t_{m}\right)=\left(t_{2}-1\right) \cdots\left(t_{m-1}-1\right) \Delta\left(t_{1}, t_{2}\right)
$$

where $\Delta\left(t_{1}, t_{2}\right)$ is one of the examples from $3 \mathrm{~B}$ or $3 \mathrm{C}$, and hence cannot be the Alexander polynomial of a two-component link with linking number $b$. (Thus, $b \neq 0,1,2$.) In Theorem 4.3 it will be shown that $f_{m}$ cannot be the Alexander polynomial of any $m$-component link with linking matrix $B$.

For $m>2$, the Torres conditions are

T1

$$
\Delta\left(t_{1}, \ldots, t_{m}\right)=(-1)^{m} t_{1}^{a_{1}} \cdots t_{m}^{a_{m}} \Delta\left(t_{1}^{-1}, \ldots, t_{m}^{-1}\right) \text { for some } a_{i}
$$

$$
\Delta\left(t_{1}, \ldots,{ }_{i}^{1}, \ldots, t_{m}\right)=\left(t_{1}^{h_{1}} \cdots \hat{t}_{i} \cdots t_{m}^{h_{m i}}-1\right) \Gamma_{i}
$$

where $\Gamma_{i}$ satisfies Torres' conditions for $m-1$ variables.

(4.1) LemMA. The polynomial $f_{3}\left(t_{1}, t_{2}, t_{3}\right)$ determines the linking matrix $B$.

Proof. Suppose $B=\left(b_{i j}\right)$. We use Torres' second conditions on $f\left(t_{1}, t_{2}, t_{3}\right)$ to determine the $b_{i j}$.

$$
f_{3}\left(t_{1}, t_{2}, t_{3}\right)=\left(t_{2}-1\right)\left[\frac{1-\left(t_{1} t_{2}\right)^{h}}{t-t_{1} t_{2}} \lambda\left(t_{1}\right)-\left(1-t_{1}\right)\left(1-t_{2}\right)\left(\frac{1-\left(t_{2} t_{2}\right)^{b-1}}{1-t_{1} t_{2}}\right) m\right] \text {, }
$$

where $\lambda\left(t_{1}\right)=\lambda\left(t_{1}^{-1}\right), \lambda(1)=1$ and $m \in \mathbf{Z}$. Then

(i) by substituting $t_{1}=1$, we get

$$
t_{2}^{b}-1=\left(t_{2}^{b_{12}} t_{3}^{b_{13}}-1\right) \Gamma_{1},
$$

for $\Gamma_{1}$ satisfying Torres' conditions for two variables. Clearly $b_{13}=0$, and $b_{12} \neq 0$ since $b \neq 0$. There are two choices for $b_{12}$ which are considered below.

(ii) by substituting $t_{2}=1$, we get $f\left(t_{1}, 1, t_{3}\right)=0$. Hence $\Gamma_{2}=0$, or $b_{12}=b_{23}=0$, which is impossible. 
(iii) by substituting $t_{3}=1$, we get

$$
\left(t_{2}-1\right)\left[\frac{1-\left(t_{1} t_{2}\right)^{h}}{1-t_{1} t_{2}} \lambda\left(t_{1}\right)-\left(1-t_{1}\right)\left(1-t_{2}\right)\left(\frac{1-\left(t_{1} t_{2}\right)^{h-1}}{1-t_{1} t_{2}}\right) m\right]=\left(t_{2}^{h_{23}}-1\right) \Gamma_{3}
$$

for some $\Gamma_{3}$ satisfying Torres' conditions for two variables. (Recall $b_{13}=0$.)

Now consider the cases for $b_{12}$.

Case I. $b_{12}$ is a proper divisor of $b$ or $b_{12}=1$. Then $\Gamma_{1}=1+t_{2}^{h_{12}}+\cdots+t_{2}^{h-b_{12}}$, so $\Gamma_{1}(1,1)=b_{23}>1$. Then in (iii)

$$
\left(t_{2}^{b_{23}}-1\right) \Gamma_{3}=\left(t_{2}-1\right)\left[\frac{1-\left(t_{1} t_{2}\right)^{b}}{1-t_{1} t_{2}} \lambda\left(t_{1}\right)-\left(1-t_{1}\right)\left(1-t_{2}\right)\left(\frac{1-\left(t_{1} t_{2}\right)^{b-1}}{1-t_{1} t_{2}}\right) m\right] \text {. }
$$

That is,

$$
\begin{aligned}
(1+ & \left.t_{2}+\cdots+t_{2}^{h_{23}-1}\right) \Gamma_{3} \\
& =\left[\frac{1-\left(t_{1} t_{2}\right)^{h}}{1-t_{1} t_{2}} \lambda\left(t_{1}\right)-\left(1-t_{1}\right)\left(1-t_{2}\right)\left(\frac{1-\left(t_{1} t_{2}\right)^{h-1}}{1-t_{1} t_{2}}\right) m\right] .
\end{aligned}
$$

Thus, $1+t_{2}+\cdots+t_{2}^{b_{23}-1}$ divides the right-hand side.

Let $t_{2}=1$; then $b_{23}$ divides $1+t_{1}+\cdots+t_{1}^{b-1}$, which is impossible since $b_{23}>1$.

Case II. $b_{12}=b$. Then $\Gamma_{1}=1$ and $\Gamma_{1}(1,1)=b_{23}=1$. Thus $\left(t_{2}-1\right)$ divides $f\left(t_{1}, t_{2}, 1\right)$, which is clearly true, and

$$
B=\left[\begin{array}{lll}
0 & b & 0 \\
b & 0 & 1 \\
0 & 1 & 0
\end{array}\right]
$$

(4.2) Lemma. Up to a permutation of $\{3,4, \ldots, m-1\}$, the polynomial $f_{m}\left(t_{1}, \ldots, t_{m}\right)$ determines the linking matrix $(m \geqslant 3)$.

Proof. (Induction on $m$. The first step is (4.1).)

(i) Substituting $t_{1}=1$ gives

$$
\left(t_{2}^{h}-1\right)\left(t_{3}-1\right) \cdots\left(t_{m-1}-1\right)=\left(t_{2}^{b_{12}} \cdots t_{m}^{h_{1 m}}-1\right) \Gamma_{1} .
$$

Clearly $b_{1 m}=0$. The choices for $b_{1 j}, 2 \leqslant j \leqslant m-1$, are considered below.

(ii) Substituting $t_{j}=0,2 \leqslant j \leqslant m-1$, gives

$$
f_{m}\left(t_{1}, 1, t_{3}, \ldots, t_{m}\right)=\cdots=f_{m}\left(t_{1}, \ldots, t_{m-2}, 1, t_{m}\right)=0 .
$$

(iii) Substituting $t_{m}=1$ gives

$$
\begin{aligned}
f_{m}\left(t_{1}, \ldots, t_{m-1}, 1\right) & =\left(t_{2}-1\right) \cdots\left(t_{m-1}-1\right) \Delta\left(t_{1}, t_{2}\right) \\
& =\left(t_{2}^{h_{2}} \cdots t_{m-1}^{h_{m}}-1\right) \Gamma_{m} .
\end{aligned}
$$

Now consider the choices for $b_{1 j}, 2 \leqslant j \leqslant m-1$.

Case I. $b_{12}=0$. By permuting $\{3, \ldots, m-1\}$ if necessary, we may assume $b_{1, m-1}=1, b_{i j}=0,3 \leqslant j \leqslant m-2$, the first row of $B$ is $\left(\begin{array}{llll}0 \_ & 1 & 0\end{array}\right)$ and

$$
\Gamma_{1}\left(t_{2}, \ldots, t_{m}\right)=\left(t_{2}^{h}-1\right)\left(t_{3}-1\right) \cdots\left(t_{m-2}-1\right) .
$$


Then

$$
\begin{aligned}
\Gamma_{1}\left(t_{2}, \ldots, t_{m-1}, 1\right) & =\left(t_{2}^{b}-1\right)\left(t_{3}-1\right) \cdots\left(t_{m-2}-1\right) \\
& =\left(t_{2}^{h_{2 m}} \cdots t_{m-1}^{h_{m-1}}-1\right) \Gamma_{1 m} .
\end{aligned}
$$

Hence $b_{m-1,1}=0$. There are three choices for $b_{2 m}$. If $b_{2 m}=b$, then $b_{j m}=0$, $3 \leqslant j \leqslant m-2$, and in (iii) above $f_{m}\left(t_{1}, \ldots, t_{m-1}, 1\right)$ must be divisible by $\left(t_{2}^{b}-1\right)$ which cannot happen. If $b_{2 m}=1$, then $b_{j m}=0,3 \leqslant j \leqslant m$, the last column of $B$ is (10 10 $0)^{\mathrm{tr}}$ and

$$
\Gamma_{1 m}\left(t_{2}, \ldots, t_{m-1}\right)=\left(t_{3}-1\right) \cdots\left(t_{m-2}-1\right)\left(1+t_{2}+\cdots+t_{2}^{b-1}\right) .
$$

Note that $\Gamma_{1 m}$ is the same as $\Gamma_{1}$ of Case II with one fewer variable. Following the procedure of Case II, one finds that $b_{m-2, m-1}=b$ which leads to a contradiction, namely that $\left(t_{m-2}^{h}-1\right)$ divides $\Gamma_{1 m}\left(1, t_{2}, \ldots, t_{m-1}\right)$.

Case II. $b_{12}=1$ or $b_{12}$ is a proper divisor of $b$. Then $b_{1 j}=0$ for $3 \leqslant j \leqslant m-1$, the first row of $B$ is $\left(\begin{array}{llll}0 & b_{12} & 0\end{array}\right)$ and

$$
\Gamma_{1}\left(t_{2}, \ldots, t_{m}\right)=\left(1+t_{2}^{b_{12}}+\cdots+t_{2}^{b-b_{12}}\right)\left(t_{3}-1\right) \cdots\left(t_{m-1}-1\right) .
$$

Thus

$$
\begin{aligned}
\Gamma\left(1, t_{3}, \ldots, t_{m}\right) & =\frac{b}{b_{12}}\left(t_{3}-1\right) \cdots\left(t_{m-1}-1\right) \\
& =\left(t_{3}^{h_{23}} \cdots t_{m}^{h_{2 m}}-1\right) \Gamma_{12} .
\end{aligned}
$$

Clearly $b_{2 m}=0$. By permuting $\{3, \ldots, m-1\}$ if necessary, we may assume $b_{23}=1$ and $b_{2 j}=0,4 \leqslant j \leqslant m-1$, and the second row of $B$ is $\left(\begin{array}{lllll}1 & 0 & 1 & 0\end{array}\right.$ $0)$. Continuing this way

$$
\begin{aligned}
& \Gamma_{12}\left(t_{3}, \ldots, t_{m}\right)=\frac{b}{b_{12}}\left(t_{4}-1\right) \cdots\left(t_{m-1}-1\right) \\
& \vdots \\
& \Gamma_{12 \cdots m-2}\left(t_{m-1}, t_{m}\right)=\frac{b}{b_{12}} \text { so } b_{m-1, m}=\frac{b}{b_{12}}>1
\end{aligned}
$$

and

$$
B=\left[\begin{array}{ccc}
0 & \frac{b}{b_{12}} \\
& \frac{b}{b_{12}} &
\end{array}\right]
$$

But then

$$
\begin{aligned}
\Gamma_{1}\left(t_{2}, \ldots, t_{m-1}, 1\right) & =\left(t_{3}-1\right) \cdots\left(t_{m-1}-1\right)\left(1+t_{2}^{h_{12}} \cdots t_{2}^{b-h_{12}}\right) \\
& =\left(t_{m-1}^{h / h_{12}}-1\right) \Gamma_{1 m}
\end{aligned}
$$


(since $\left.b_{j m}=0, j<m-1\right)$. This is impossible (since $b / b_{12}>1$ so $\left(t_{m-1}^{h / h_{12}}-1\right)$ does not divide $\left(t_{3}-1\right) \cdots\left(t_{m-1}-1\right)\left(1+t_{2}^{b_{12}} \cdots t_{2}^{h-h_{12}}\right)$.

Case III. $b_{12}=b$. Then, $b_{i j}=0,3 \leqslant j \leqslant m-1$. Hence the first row of $B$ is $(0$ b 0 $0)$, and

$$
\begin{aligned}
\Gamma_{1}\left(t_{2}, \ldots, t_{m}\right) & =\left(t_{3}-1\right) \cdots\left(t_{m-1}-1\right) \\
& =\left(t_{2}^{h_{2}} \cdots t_{m-1}^{h_{m}} 1^{1, m}-1\right) \Gamma_{1 m} .
\end{aligned}
$$

Clearly $b_{2 m}=0$. By permuting $\{3, \ldots, m-1\}$ if necessary, we may assume $b_{m-1, m}$ $=1$ and $b_{j m}=0,3 \leqslant j \leqslant m-2$. Hence, the last column of $B$ is $\left(\begin{array}{llll}0 & 0 & 1 & 0\end{array}\right)^{\mathrm{tr}}$, and $\Gamma_{1 m}=\left(t_{3}-1\right) \cdots\left(t_{m-2}-1\right)$. Continuing in this way we find

$$
B=\left[\begin{array}{lll}
0 & b & 0 \\
b & 0 & 1 \\
0 & 1 & 0 \\
& &
\end{array}\right]
$$

and in (iii) above $\Gamma_{m}=f_{m-1}\left(t_{1}, \ldots, t_{m-1}\right)$, and in (ii) $\Gamma_{2}=\cdots=\Gamma_{m-1}=0$.

(4.3) THEOREM. With the conditions as above $f_{m}\left(t_{1}, \ldots, t_{m}\right)$ cannot be the Alexander polynomial of an m-component link with linking matrix $B$.

Proof (Induction on $m$ ). For $m=3, f_{3}\left(t_{1}, t_{2}, 1\right)=\left(t_{2}-1\right) \Delta\left(t_{1}, t_{2}\right)$. Hence $\Gamma_{3}=\Delta\left(t_{1}, t_{2}\right)$, which is not allowed by Torres' second condition.

For $m>3$,

$$
f_{m}\left(t_{1}, \ldots, t_{m-1}, 1\right)=\left(t_{m-1}-1\right)\left[\prod_{i=2}^{m-2}\left(t_{i}-1\right)\right] \Delta\left(t_{1}, t_{2}\right) .
$$

Hence

$$
\Gamma_{m}=\prod_{i=2}^{m-2}\left(t_{i}-1\right) \Delta\left(t_{1}, t_{2}\right)=f_{m-1}\left(t_{1}, \ldots, t_{m-1}\right),
$$

which is not allowed by induction.

\section{REFERENCES}

[B] J. H. Bailey, Alexander incariants of links, Ph.D. thesis, Univ. of British Columbia, 1977.

[H] J. A. Hillman. The Torres conditions are insufficient, Math. Proc. Cambridge Philos. Soc. 89 (1981). $19-22$.

[J] N. Jacobson, Basic algehra. I, Freeman, San Francisco, Calif., 1974. p. 267.

[La] S. Lang. Algehra, Addison-Wesley, Reading, Mass., 1965.

[La-1] _. Algehraic numbers, Addison-Wesley, Reading, Mass.. 1964.

[L-1] J. P. Levine, A method for generating link polynomials. Amer. J. Math. 89 (1967), 69-84.

[L-2] _. Structure of knot modules, Lecture Notes in Math., Vol. 772, Springer-Verlag, New York, 198(). pp. $90 \mathrm{ff}$.

[M] D. A. Marcus, Number fields, Springer-Verlag, New York, 1977.

[S] J. P. Serre, Local fields, Springer-Verlag. New York, 1979, p. 11

[Z] H. Zassenhaus, The theory of groups, Chelsea, New York, 1958, p. 90.

Dipartilint of Mathematics. Tufts Univirsity, Midford, Massachusetts 02155 